\title{
La paridad del poder adquisitivo: nueva evidencia para Colombia y Latinoamérica*
}

\section{Purchasing Power Parity: \\ another look to the evidence from Colombia and Latin America}

Orlando Mauricio Lorduy Herrera**

Recibido: 28 de enero de 2015

Revisado: 5 de febrero de 2015

Aprobado: 16 de marzo de 2015

\section{Resumen}

El problema de la relación entre los precios y los tipos de cambio en la economía internacional ha transcendido el tiempo muy a pesar de que a nivel teórico existe una tendencia generalizada a creer que dicha relación es una ley económica fundamental derivada de la ley de un solo precio, y que es conocida como la paridad del poder adquisitivo (PPA). En este trabajo se hace una nueva revisión empírica del cumplimiento de la PPA para Colombia en comparación con algunos países latinoamericanos de los que se dispone de información suficiente (Argentina, Brasil, Chile, Ecuador, México, Perú

* Como citar este articulo: Lorduy, O. (2014). La paridad del Poder adquisitivo, nueva evidencia para Colombia y Latinoamérica. Revista CIFE, 16 (25), 123-152.

** Docente de la Universidad Pontificia Bolivariana Seccional Montería. Economista, magíster en Ciencias Económicas. Correo electrónico: orlando.lorduy@upb.edu.co 
y Venezuela). El periodo de referencia va desde 1970 hasta 2011 con datos mensuales. Se utilizan las técnicas más recientes para el análisis de cointegración en panel de datos. Los resultados muestran que existe evidencia de la presencia de una relación de paridad de largo plazo para estas economías que, en el papel, son bastante similares en cuanto a estructura económica e idiosincrasia.

Palabras clave: Ley de un solo precio, paridad del poder adquisitivo, tasa de cambio, cointegración en panel de datos.

Clasificación JEL: F41, F42, C23

\begin{abstract}
The problem of the relation between the prices and the exchange rates in the international economy has transcended time, very in spite of the fact that theoretically there is a widespread tendency to believe that this is an economic fundamental law derived from the Law of One Price, and that is known as the Purchasing Power Parity (PPP). This paper presents a new empirical review of the fulfillment of the PPA for Colombia, in comparison with some Latin-American countries for which sufficient information is available (Argentina, Brazil, Chile, Ecuador, Mexico, Peru y Venezuela). The reference period runs from 1970-2011, with monthly data; this one uses the most resent techniques for cointegration in data panel. The results show that there exists evidence of the presence of a long term parity relationship for these economies which, on paper, are quite similar in terms of economic structure and idiosyncrasy.
\end{abstract}

Keywords: Law of one price, Purchasing power parity, exchange rate, panel cointegration.

Classification JEL: F41, F42, C23 


\section{Introducción}

Uno de los acertijos ${ }^{1}$ fundamentales que ha abordado la economía internacional en tiempos no muy lejanos tiene que ver con el cumplimiento de la teoría de la paridad del poder adquisitivo (PPA), formalizada por el economista sueco Gustav Cassel en 1916. En pocas palabras, dicha teoría se refiere a la relación directa que existe entre los precios de los bienes transables, dados los tipos de cambio, y en ausencia de barreras naturales y artificiales. Si bien el autor reconocía que se pueden presentar desviaciones de corto plazo que impidan el cumplimiento de la PPA, la ortodoxia económica ha mantenido la creencia de que, en condiciones ideales, dicha relación es una ley económica primordial (Dornbusch, 1988, p. 1077).

La PPA deriva de la ley de un solo precio y, como tal, implica que las cestas de bienes idénticas valoradas en una moneda común deben tener el mismo precio dadas ciertas condiciones. Se entiende, entonces, que las variaciones de los precios relativos tienen efectos momentáneos en las tasas de cambio y viceversa, solamente hasta que se alcance el ajuste propuesto por la teoría. En la práctica, la evidencia muestra que las tasas de cambio son altamente volátiles en periodos cortos, por lo cual, las autoridades monetarias y cambiarias tienen un especial interés en la determinación de esta relación, principalmente con fines de establecer qué tan competitiva puede ser una economía en un determinado momento.

La tasa de cambio real (TCR) se ha constituido en uno de los indicadores de competitividad más utilizado por los países. La observación de la evolución histórica de dicha variable no hace más que reafirmar las dudas con respecto al cumplimiento de la PPA, y los desarrollos investigativos más recientes intentan descubrir el porqué de sus desviaciones incluso en periodos prolongados. Este trabajo se realiza, precisamente, como una contribución al debate para Colombia y Latinoamérica; se busca entregar elementos adicionales que permitan entender mejor el problema de la PPA, respondiendo al interrogante de si se ha cumplido dicha relación para el periodo posterior a Bretton Woods, utilizando como variables fundamentales las TCR calculadas por el Banco de la República para un grupo de economías latinoamericanas con referencia a Colombia.

Este trabajo está dividido en cuatro secciones, además de esta introducción. En la primera se presentan los referentes teóricos, incluyendo una revisión de los hechos estilizados para la PPA, y una exploración a los principales modelos empíricos utilizados para contrastar esta relación; en la segunda se revisa el estado del arte para Colombia; en la tercera se describe la metodología y se entregan los resultados de la investigación; por último, en la cuarta sección se recogen las conclusiones y recomendaciones.

1 La palabra utilizada por Rogoff (1996), Obstfeld \& Rogoff (2000) es puzzle, y describe problemas de la economía internacional sin aparente solución empírica. 


\section{La paridad del poder adquisitivo (PPA)}

Según Dornbusch (1988, p. 1077), el concepto moderno de la PPA se puede asociar con los trabajos realizados por el economista sueco Gustav Cassel en los albores de la Primera Guerra Mundial y el subsiguiente periodo de entreguerras. Ya en 1916, Cassel bautizó la relación entre "tasas de cambio" y "niveles de precios" con el nombre con el que actualmente se conoce: Purchasing Power Parity o PPP; no obstante, el autor reconoció desde un primer momento la posibilidad de que existan desviaciones en el comportamiento de las tasas de cambio y los precios por motivos que serán expuestos más adelante en esta sección.

Este concepto deriva directamente de la ley de un solo precio, la cual plantea que, si dos economías tienen nexos comerciales entre sí, y se supone que no existen barreras artificiales al comercio (aranceles, licencias, cuotas, etc.), y que, además, los costos de transporte son irrelevantes ${ }^{2}$, se puede pensar en que los productos idénticos que se comercien entre ellas tendrán el mismo precio en términos de una moneda común. Formalmente, la ley implica que:

$$
P_{i}=\operatorname{tcn} P_{i}^{*}(1)
$$

$P_{i}$ es el precio del bien expresado en moneda local y vendido en el país; $P_{i}^{*}$ es el precio del mismo bien, pero expresado en moneda extranjera y vendido en el país extranjero; y tcn es la tasa de cambio nominal de la moneda local en términos de la moneda extranjera.

El arbitraje es el proceso que asegura que la ley de un solo precio se cumpla, dado que ninguno de los países participantes en el comercio internacional puede obtener ganancias extraordinarias siempre que los bienes transados sean homogéneos. En términos más formales, los excedentes del mercado solamente están influenciados por los cambios de las cantidades transadas y no por cambios de los precios relativos del bien en consideración.

La teoría de la PPA es una generalización de la ley de un solo precio; dadas las condiciones de comercio planteadas anteriormente, una cesta o canasta ponderada de bienes tiene la misma valoración entre dos países en términos de una misma moneda, lo que implica que:

$$
P=\operatorname{tcn} P^{*}(2)
$$

2 Siguiendo a Dornbusch (1993, pp. 102-104), esto es equivalente a la no existencia de barreras naturales, las cuales refieren a costos de transporte excesivamente altos y/o al tiempo que lleva transportarlos, que impide que algunos bienes específicos conserven sus características al llegar a su destino. En ambos casos se estará en la frontera de la transabilidad y la no transabilidad. 
$\mathrm{O}$, alternativamente:

$$
\text { tcn }=P / P^{*}\left(2^{\prime}\right)
$$

$P$ y $P^{*}$ representan las cestas de bienes idénticas valoradas en la moneda local de cada país, respectivamente ${ }^{3}$. De nuevo, los índices de precios escogidos deben ser iguales cuando se expresan en una moneda común o, en otras palabras, en las tasas de cambio vigentes, el poder adquisitivo interno y externo de cada moneda es siempre el mismo. Es por esto que a la teoría de la PPA también se le conoce como la "teoría de la inflación de las tasas de cambio" (Op. cit. p. 1075).

De lo anterior es posible deducir que, si la ley de un solo precio se cumple para n bienes, también debe cumplirse la PPA, dado que las cestas de referencia son las mismas ${ }^{4}$. Una importante implicación derivada del arbitraje y de la homogeneidad de las cestas de bienes es que la ecuación (2') se puede reescribir como una relación unitaria:

$$
1=P / \operatorname{tcn} P_{*}\left(2^{\prime \prime}\right)
$$

dado que $P$ / $P^{*}=C$; $C$ representando una constante. Este resultado tiene importantes consecuencias cuando se introduce el concepto más específico de tasa de cambio real (TGR), como se detallará en el apartado 2.1.

A la ecuación (2') se le suele llamar la versión "fuerte" o "absoluta" de la PPA por las características de los supuestos que se deben cumplir; existe una versión que relaja los supuestos de la versión fuerte, y se conoce como la versión "débil" o "relativa", cuya expresión matemática se puede plantear como sigue:

$$
\Delta t c n / t c n_{t-1}=\Delta P / P_{t-1}-\Delta P^{*} / P_{t-1}^{*}(3)
$$

Esta ecuación representa las variaciones porcentuales $(\Delta)$ de la tcn de los precios domésticos (tasa de inflación interna, $\pi$ ) y de los precios externos (tasa de inflación externa, $\pi^{*}$ ), respectivamente. Por último, se llega a la siguiente expresión en términos de relaciones de cambio:

$$
\Delta t c n / t c n_{t-1}=\pi_{t}-\pi_{t}^{*}\left(3^{\prime}\right)
$$

Definida para un intervalo de tiempo cualesquiera, la versión relativa de la PPA implica que las variaciones en las tcn se deben a los desajustes de los niveles de precios de los países.

3 Con $P=\Delta\left(p_{1}, \ldots, p_{\mathrm{i}}, \ldots, p_{\mathrm{n}}\right)$ y $\Delta P^{*}=\left(p_{1}, \ldots, p_{\mathrm{i}}, \ldots, p_{\mathrm{n}}\right), \mathrm{n} \geq 1$, funciones homogéneas de grado 1.

4 Krugman \& Obstfeld (2003, p. 391) reconocen, al igual que muchos otros teóricos, que aun cuando la ley de un solo precio "no se cumple literalmente, las fuerzas económicas que operan detrás de ella ayudarán con el tiempo a igualar el poder adquisitivo de una moneda con el de las demás divisas". 
Dichos niveles de precios no necesariamente se recogen por medio de cestas idénticas comparables entre países, sino, más bien, cada país escoge sus bienes y servicios representativos según las preferencias y el ingreso de sus consumidores.

La diferenciación entre la PPA absoluta y la PPA relativa es importante para efectos prácticos, dado que el concepto original (absoluto) implica restricciones para su contraste al exigir la construcción de índices que utilicen cestas idénticas en cuanto a bienes y ponderaciones para un periodo relativamente $\operatorname{largo}^{5}$. Pero aun si se obtuviera la información para construir dichos índices, siempre existirá el problema que representa la existencia de los bienes no transables, no tanto por ellos mismos como tales, sino más bien por su uso como parte del proceso productivo de los bienes transables.

\subsection{La tasa de cambio real (TCR) y la teoría de largo plazo}

En la práctica, la evidencia empírica demuestra que la tcn presenta grandes fluctuaciones en periodos relativamente cortos, por lo cual se hace necesario definir una relación que recoja los movimientos tanto de dicha tasa nominal como de los precios de las economías comparadas. En ese orden de ideas, se introduce el concepto de tasa de cambio real, como sigue:

$$
T C R=\operatorname{tcn} P^{*} / P(4)
$$

Tal como está definida la TCR, permite comparar el poder adquisitivo de cestas de bienes más que de monedas, por lo que es de amplia utilización en los estudios de paridad como indicador de competitividad de las economías ${ }^{6}$. Dado que los desajustes de $t c n, P$ y $P^{*}$ no tienen por qué ser simétricos, el proceso de retorno al equilibrio de la TCR dependerá de los tiempos de ajuste de cada una de las variables implicadas (cambios nominales). Teóricamente esto significa que, cuando $P$ y/o $P^{*}$ cambian, la tcn fluctúa de tal forma que la TCR permanece constante o, al menos, no cambia permanentemente.

Por supuesto, una variación de la TCR representa una desviación de la PPA relativa (transformando la ecuación (4) en términos de variaciones porcentuales). Dicha desviación puede darse siempre que el tiempo de ajuste de las variables difiera de manera sustancial ${ }^{7}$. Para este trabajo se toma como referente la ecuación cuatro, la cual se constituye en un instrumento válido no solamente para contrastar las relaciones planteadas

5 Teniendo en cuenta que la PPA es una teoría de largo plazo, cualquier intento por demostrarla requiere de la disposición de series estadísticas relativamente extensas (ver Froot \& Rogoff, 1994).

6 Para Colombia se pueden identificar como los principales trabajos relacionados los de Huertas y Oliveros (2003), Echavarría et al. (2005), Huertas (2005) y Alonso et al. (2008).

7 De hecho, es bastante probable que las desviaciones de la PPA sean permanentes y no transitorias por efecto de factores no monetarios presentes en todas las economías (por ejemplo, cambios en la productividad de los factores, en la tenencia de activos en el exterior, en los términos de intercambio, etc.). 
por la PPA, sino también para determinar el desalineamiento de los precios frente a la tasa de cambio como medida de competitividad.

\subsection{Modelos empíricos para la PPA}

Se pueden distinguir al menos cuatro etapas probatorias para validar la teoría de la PPA empíricamente:

1. Una primera etapa en la que se utilizan las pruebas más antiguas que buscaban comprobar simplemente que la PPA se cumple.

2. Una segunda etapa en la que el análisis de series de tiempo gana preponderancia; se intentaba contrastar la hipótesis nula de si la PPA tiene validez para periodos suficientemente largos.

3. La tercera etapa avanza en la utilización de pruebas de cointegración que buscan demostrar la existencia de perturbaciones que afectan permanentemente la PPA (relaciones de largo plazo).

4. La última etapa tiene que ver con el uso de las metodologías de cointegración en análisis de panel de datos con el fin de maximizar el poder de predicción de los test econométricos más recientes.

De estas etapas, las dos primeras han sido suficientemente estudiadas en numerosos trabajos, por lo que aquí se describen de manera muy sucinta. La primera forma que se utilizó para la validación empírica de la PPA se refiere al contraste de su versión fuerte ${ }^{8}$. El siguiente ejercicio consistió en confrontar la versión débil de la PPA, cuyo planteamiento econométrico se puede escribir como sigue:

$$
\Delta p_{t}=\Delta p_{t}^{*}+\Delta t c n_{t}(5)
$$

Las variables $p_{t}, p_{t}^{*}$ y $t n_{\mathrm{t}}$ (expresadas en logaritmos) vienen representadas por medio del $I P C, I P C^{*}$ y la $t c n$, respectivamente. El subíndice t representa la dimensión temporal de las variables.

Los resultados de estos análisis iniciales estaban condicionados por la ausencia de instrumentos estadísticos y econométricos que permitieran distinguir entre efectos de corto plazo y efectos de largo plazo, de esta manera no era posible tener en cuenta la existencia de shocks no monetarios que posiblemente afectaran la senda de equilibrio de la $\mathrm{PPA}^{9}$.

8 No está de más especificar que son pocos los autores que se han aventurado a construir índices usando cestas de bienes y servicios idénticas; el trabajo más conocido e influyente fue realizado por Summers \& Heston en 1991.

9 Un defecto fundamental de estas primeras estimaciones era que no podían explicar la no estacionariedad de las variables mediante la realización de regresiones por el método de mínimos cuadrados ordinarios; en otros términos, si los residuos no son estacionarios, la relación entre las variables endógenas y exógenas se inválida. 
En etapas intermedias de desarrollo de los instrumentos econométricos surgió una segunda generación de pruebas para la validación de la PPA. En este caso se buscaba contrastar la hipótesis de que la tasa de cambio real sigue un paseo aleatorio o, en su defecto, la PPA prevalece en el largo plazo. Se asumía que la relación entre precios internos y externos se cumple en términos de una misma moneda, con el fin de comprobar la hipótesis:

$$
T C R_{t} \equiv t c n_{t}-p_{t}+p_{t}^{*}(6)
$$

En la ecuación, $T C R_{t}$ (en logaritmos) es estacionaria. Estos modelos tenían un bajo poder estadístico que hacía imposible afirmar si era adecuado rechazar o no la hipótesis; teóricamente, la volatilidad de las tasas de cambio no permite afirmar que exista un paseo aleatorio, especialmente si se consideran periodos en los cuales las tasas de cambio han sido flexibles (Froot \& Rogoff, 1994, pp. 6-7). El perfeccionamiento de las pruebas de estacionariedad por medio de los aportes de Dickey \& Fuller (1979) jugó un papel importante en la evolución de los modelos de segunda generación. Bajo la hipótesis de comportamiento tipo random walk se utilizaron modelos en los que se pueden incorporar constantes de proporcionalidad y variables de tendencia. El principal problema de los métodos de segunda generación es que existen dificultades para rechazar la hipótesis nula de raíz unitaria cuando se comparan países industrializados con datos mensuales y tasas de cambio flotantes (Meese \& Rogoff (1985), p. 19); sin embargo, los resultados no son concluyentes para países que tienen tasas de cambio fijas (Froot \& Rogoff, 1994, p. 8).

La tercera generación de modelos y pruebas para la PPA incluye la utilización de métodos de cointegración para determinar relaciones de equilibrio de largo plazo entre variables cuyos mecanismos de ajuste no están especificados, obviando el problema de endogeneidad de las variables (Ibíd.; Sarno \& Taylor, 2002, p. 77). El modelo básico toma la siguiente forma:

$$
t c n_{t}=\gamma p_{t}+\gamma^{*} p_{t}^{*}+\mu_{t}(7)
$$

En la ecuación, $\gamma$ y $\gamma^{*}$ son los coeficientes de proporcionalidad, en tanto $\operatorname{tcn}_{\mathrm{t}} \mathrm{y} \mu_{\mathrm{t}}$ deben ser estacionarias si $\gamma=\gamma^{*}=1$. Sin embargo, lo más probable es que estos no tomen dicho valor, por ejemplo, si existe tendencia en los precios relativos de los transables y de los no transables, si los precios de las canastas de bienes transables se modifican permanentemente respecto a los precios de los no transables (por cambios de la productividad, por ejemplo); entonces se producirá una variación permanente de los precios relativos. Lo mismo puede ocurrir si los precios que se modifican corresponden a los bienes no transables, por ejemplo, por cambios de gustos o del ingreso permanente (Froot \& Rogoff, 1994, p. 19).

Las primeras aplicaciones de los métodos de cointegración para contrastar la PPA siguen tres pasos básicos. Primero se realizan pruebas $\mathrm{ADF}^{10}$ a las variables (tcn, $p$ y $p^{*}$ o,

10 Prueba Dickey - Fuller aumentada, para determinar si las series son estacionarias; en caso de que no lo sean, debe establecerse su orden de integración. 
en su defecto, a los precios relativos; en el primer caso, se trata de un modelo trivariado, mientras que el segundo es un modelo bivariado). Asumiendo que alguna de las variables no sea estacionaria, el segundo paso consiste en estimar la ecuación de cointegración (8) usando el método de mínimos cuadrados ordinarios si se sigue la metodología de Engle \& Granger (1987), o bien, utilizando una especificación de máxima verosimilitud en el caso de que se utilice la metodología de Johansen (1988). El tercer paso implica la realización de la prueba $\mathrm{ADF}$ a los residuos de dicha ecuación para determinar si estos son estacionarios, en cuyo caso se dice que las series están cointegradas ${ }^{11}$ (Ibíd., p. 20).

Si bien los resultados de esos modelos superan a los alcanzados por los más antiguos, estos varían considerablemente cuando las tasas de cambio son flexibles, dado que la hipótesis nula de que no existe relación de cointegración se rechaza más en periodos de tasas de cambio fijas que en periodos de tasas flexibles. Un hallazgo adicional encuentra que, para el caso de series pos Bretton Woods, la hipótesis nula se rechaza más frecuentemente en sistemas trivariados que en sistemas bivariados. Finalmente, se observa que las estimaciones de $\gamma$ y $\gamma^{*}$ pueden diferir ampliamente de un estudio a otro cuando se estudian periodos más recientes, lo que conlleva a que la interpretación económica de los fenómenos sea más compleja (Ibíd., p. 21; Sarno \& Taylor (2002, p. 78).

La última etapa de test para validar la PPA en realidad es una ampliación de la anterior. Las investigaciones más recientes en la materia se sustentan en la aplicación de pruebas de cointegración para las variables agrupadas en forma de panel. Al igual que en la etapa previa, se busca determinar la existencia de relaciones estables de largo plazo para los precios y los tipos de cambio nominales o, en su defecto, para las tasas de cambio reales. Una especificación típica para contrastar la PPA en su versión fuerte viene dada por la siguiente expresión:

$$
t c n_{i t}=\alpha_{i}+\beta_{i} p_{i t}+\mu_{i t}(8)
$$

En la ecuación, ahora, además de la dimensión temporal $(t)$, también se incluye la dimensión trasversal (i), que se refiere al número de unidades sociales incluidas en el panel, en este caso, los países seleccionados (Pérez, 2008, pp. 273-275). La variable $p_{i t}$ corresponde al logaritmo del diferencial de precios entre el $i$-ésimo país y el país de referencia en el periodo $t$. Autores como Taylor (2000b), Azali et al. (2001), Pedroni (2001), Imbs et al. (2005), Jenkins \& Snaith (2005), Chortareas \& Kapetanios (2009), Al Omar \& Ghali (2009) han utilizado estos nuevos instrumentos de contraste desarrollados en las últimas dos décadas, para conseguir mayor poder de predicción, con resultados favorables, aunque no unánimes.

11 Si las desviaciones de la PPA son temporales, tal como supone la teoría, entonces el término de error $\mu \_t$ debe ser estacionario; lo anterior garantiza que a lo largo del tiempo se consiga la reversión a la media de las variables que forman parte de la ecuación (8). 
En conclusión, a pesar de los avances alcanzados en cuanto a metodologías estadísticas y disponibilidad de series de tiempo, no se puede afirmar plenamente que la teoría de la PPA sea un hecho empírico incontrovertible, sino, más bien, se deben seguir explorando posibilidades para alcanzar conclusiones más acertadas y aportar el debate académico. Para efectos de esta investigación se siguen los estándares establecidos por los métodos de cuarta generación, tal como se describe en la nota metodológica.

\section{Evidencia para Colombia}

En esta sección se resumen algunos trabajos que abordan el problema de la PPA en mayor o menor grado y en diferentes perspectivas. El primer trabajo que trata el problema de manera explícita pertenece a Carrasquilla (1989); en él se recogen las tendencias pesimistas respecto al cumplimiento de la PPA que surgieron a finales de los años setenta y durante buena parte de los años ochenta ${ }^{12}$. El autor mostró que, para el periodo de régimen de minidevaluaciones (1967-1986) (series trimestrales), la TCR presentó un componente tendencial, lo cual abre la posibilidad de la existencia de shocks reales que afectan el comportamiento de la tcn de manera permanente y, por consiguiente, el de la TCR. Por su parte, Steiner \& Wüllner (1994) demostraron la existencia de una relación de corto plazo entre inflación (usando tanto el IPG como el índice de precios al por mayor) y TCR, pero no hallaron evidencia de la existencia de una relación de largo plazo; el análisis se realizó para el periodo 1972-1992, con datos trimestrales para veinte socios comerciales de Colombia ${ }^{13}$. Ese mismo año, Meisel (1994) investigó el cumplimiento de la PPA en el largo plazo utilizando una serie construida del índice de TCR para el periodo 1924-1993; el autor encontró que la serie era integrada de orden uno, por lo cual no se pudo rechazar la hipótesis de no estacionariedad. Como siguiente paso, se estimaron pruebas de cointegración para la tasa de cambio nominal y los índices de precios interno y externo (Estados Unidos), sin que se haya encontrado evidencia de la existencia de una relación de largo plazo.

Por su parte, Rincón (2000) estimó modelos de precios para la economía colombiana tomando como periodo de estudio el comprendido entre 1980 y 1998 (series mensuales), estudiando los efectos de las variaciones de la $t c n$ sobre los precios agregados de las exportaciones, las importaciones y sobre el nivel de precios agregado. El autor se valió de técnicas de regresión multivariadas con las cuales logró tornar endógenas todas las variables en sus modelos, para encontrar que los efectos de la trasmisión de largo plazo eran incompletos; por tanto, la hipótesis de la PPA absoluta no se cumplió para dicho periodo. El siguiente trabajo corresponde a Rowland (2003), en el cual se especifican tres modelos para predecir el comportamiento de la tasa de

\footnotetext{
12 Para una visión detallada de los problemas presentados, se pueden consultar, entre otros, los trabajos de Isard (1977), Krugman (1978), Frenkel (1981) y Meese \& Rogoff (1985).

13 Arango \& Meisel (1994) criticaron este trabajo en términos metodológicos y estadísticos.
} 
cambio dólar/peso para el periodo 1970-2002 (datos trimestrales). El autor utilizó las técnicas econométricas desarrolladas por Engle \& Granger (1987) y Johansen (1988) para el tratamiento de series de tiempo en un marco multivariado estático (de corto plazo) y dinámico (de largo plazo), y comparó los resultados con los obtenidos mediante la utilización del tradicional paseo aleatorio; en la investigación se sugiere que la hipótesis de la PPA se cumplió (versión débil), siendo el modelo del paseo aleatorio el más potente en cuanto a resultados de predicción de largo plazo. También en 2003, Huertas y Oliveros buscaron estimar la TCR de equilibrio para establecer efectos de subvaluación o sobrevaluación de esta variable a través del tiempo, teniendo en cuenta la existencia de desequilibrios nominales y reales que pudieran desviar la TCR temporal o incluso permanentemente. Los autores partieron de la realización de las pruebas tradicionales para determinar el orden de integración de las series (anuales de 1958 a 2001 y trimestrales de 1980 a 2002), I(1) en la mayoría de los casos; posteriormente utilizaron pruebas de descomposición permanentetransitoria de Stock \& Watson para determinar los niveles de desequilibrio nominales (alejamiento de la PPA), y encontraron que la reversión a la media es del orden del $12 \%$ anual. Seguidamente se realizaron pruebas de cointegración para un modelo de tipo BEER (Behavioural Equilibrium Exchange Rate), las cuales sugirieron que el TGR respondía a sus fundamentales, ajustándose a una tasa aproximada del $20 \%$ anual. Finalmente, los autores advirtieron que los resultados alcanzados solo permitían hacer injerencias sobre los cambios de la media de los procesos estudiados y no de su dinámica. También en 2003 se presentó el trabajo de Oliveros \& Rowland, cuya intención era validar la hipótesis de la PPA corregida por el efecto Balassa-Samuelson (BS) para Colombia (periodo 1980 - 2002, trimestral), utilizando para ello el marco de cointegración multivariada de Johansen (1988). Los resultados alcanzados permitieron verificar la existencia de relaciones válidas, pero no perfectas, para la PPA relativa. Es destacable dentro de los resultados el hecho de que la reversión a la media de la $t c n$ resultó ser relativamente rápida, aproximadamente $11 \%$ en un trimestre.

El trabajo de Huertas (2005) buscaba determinar relaciones no lineales entre la TCR colombiana y sus fundamentales; para ello, Huertas se apoyó en técnicas econométricas de transformación para un Modelo Exponencial Autorregresivo de Transición Suave (ESTAR), con el fin de capturar movimientos no lineales entre dos periodos. Entre los resultados más relevantes se encuentra que las pruebas de raíz unitaria no lineales mostraron que las variables utilizadas eran I(1) y que, además, estaban cointegradas con la variable TCR bilateral construida para USA y para México (19832003 mensual). Por último, es de destacar que las estimaciones del modelo no lineal mostraron un mejor ajuste que las del modelo lineal, si bien ambas confirmaron la persistencia en las desviaciones de la TCR de sus niveles de equilibrio. Ese mismo año Echavarría et al. revisaron los determinantes de la TCR para Colombia bajo la concepción de tasa de equilibrio de largo plazo para el periodo posapertura (datos 
anuales 1962-2005). Según los autores, "el crecimiento de la tasa de cambio real en el muy largo plazo invalida la hipótesis de existencia de poder de paridad adquisitiva, un fenómeno común a la mayoría de países en desarrollo" (p. 7); lo anterior se puede deber a los bajos niveles de cambio técnico presentes en el sector de bienes transables, a la demanda relativa de servicios no transables y a la tendencia de la deuda externa de largo plazo. La importancia de hallar una tasa de cambio de equilibrio se encontraba en la necesidad de estabilización inherente a una política macroeconómica consistente. El trabajo concluye que los determinantes de la TCR para Colombia son el stock de activos netos, los cambios de la productividad, los términos de intercambio, los déficits fiscales y la tasa de cambio nominal.

Echavarría et al. (2008) también buscaron determinar la TCR de equilibrio para Colombia y su nivel de desalineamiento para el periodo 1962-2005; para ello utilizaron un enfoque de tendencias comunes asociadas a un Modelo de Corrección de Errores Vectorial Estructural (SVEC). Los autores encontraron que la TCR observada había estado relativamente cercana a la de equilibrio estimada, con momentos de sobrevaluación importantes y prolongados. Lo anterior parece explicarse por los efectos permanentes de largo plazo que habían tenido variables como la posición de activos externos netos, los desequilibrios de la cuenta corriente de la balanza de pagos y los shocks de comercio exterior (volumen y precios).

En el mismo sentido de los trabajos anteriores, el documento más reciente respecto a los tipos de cambio y sus diferentes alternativas de medición es el presentado por Alonso et al. (2008), en el que se parte de la definición tradicional de la TCR como medida de competitividad de una economía y en el que se exploran otras formas alternativas de medición que incluyan el efecto BS, los costos unitarios de producción como deflactores, la descomposición del IPP, entre otros. Los resultados alcanzados en este ejercicio no fueron muy diferentes de los sugeridos en cuanto a tendencia de desalineación de la TCR al usar el indicador tradicional, pero sí difirieron en lo relacionado con la magnitud de los efectos; también se pudo constatar que el efecto BS se constituye en una importante fuente de desalineamiento de la tasa de cambio frente a su valor de equilibrio.

Como se puede deducir de la revisión bibliográfica para Colombia, el enfoque de los trabajos actuales supone que la PPA se cumple parcialmente, por tanto, es necesario determinar cuáles son los fundamentales que llevan a esta situación; sin embargo, se debe hacer una observación relevante: en todos los casos, la periodicidad de las series utilizadas no es la suficiente para obtener conclusiones inapelables, por lo que es esencial seguir explorando alternativas de medición que permitan realizar aproximaciones más rigurosas, a la luz de las nuevos instrumentos estadísticos y econométricos de los que se disponen actualmente (Froot \& Rogoff (1994), Taylor (2000), Chortereas \& Kapetanios (2009), Bergin et al. (2013), entre otros). 


\section{Nota metodológica}

\subsection{Las variables}

Para la realización de este trabajo se tomaron las estadísticas de tasa de cambio real $(T C R)$, tasa de cambio nominal $(t c n)$ y de precios $(P)$ para un grupo de países latinoamericanos, de acuerdo con su disponibilidad temporal. Los datos de TCR provienen del ITGR bilateral según IPP (1970:1 - 2014:11 base 1994), calculado mensualmente por el Banco de la República. Estas series presentan una gran ventaja respecto a los índices multilaterales tradicionales, que también calcula el Banco y el FMI, ya que permiten observar la dinámica de la evolución de la competitividad en términos de cestas de bienes teniendo en cuenta las especificidades de cada economía, más aún tratándose, en este caso, de países de similares características por cercanía geográfica, idiosincrasia y, tal vez, gustos.

Por su parte, la tcn para Colombia también proviene del Banco de la República, mientras que los datos de $P$ se refieren al IPG publicado por el DANE para Colombia. Además de Colombia, se tuvieron en cuenta los siguientes países: Argentina, Brasil, Chile, Ecuador, México, Perú y Venezuela; pero solamente se obtuvieron series con la misma periodicidad para Brasil, México y Perú, lo cual merece una explicación, al menos desde el punto de vista histórico. Es bien conocida la historia de golpes de Estado y dictaduras que azotaron a muchos países latinoamericanos por varias décadas; si a eso se le suma la presencia frecuente de inflaciones altas (o de hiperinflaciones directamente), se tiene un escenario de difícil reconstrucción estadística en términos de las variables esenciales para este trabajo. Para el caso de Ecuador, la información llega hasta el año 2000, ya que en 2001 se completó el proceso de dolarización de la economía. Las series de IPC se completaron y empalmaron para todos los países mencionados, con la excepción de Brasil, cuya información está disponible desde 1994 (ver anexo 1). El panel consta de ocho secciones cruzadas no balaceadas, que comprenden un total de 4312 observaciones.

\subsection{Metodología econométrica}

Los investigadores económicos han incorporado cada vez más las técnicas de cointegración en datos de panel como instrumentos fundamentales de sus análisis de largo plazo. Existen diversas especificaciones aplicables a los modelos de panel, pertinentes según las especificidades inherentes a cada problema de investigación económica; todas ellas tienen en cuenta para su formulación que el término de error tiene tres componentes:

$$
\mu_{i t}=\tau_{i}+\theta_{t}+\varepsilon_{i t}(9)
$$

La componente $\tau_{i}$ recoge los efectos aleatorios que afectan a cada unidad social, pero que no cambian con el tiempo; por su parte, la componente $\theta_{t}$ recoge efectos temporales 
invariables entre unidades sociales; por último, la componente $\varepsilon_{i t}$ representa la parte netamente aleatoria, transversal y temporalmente de la estimación del panel. Se entiende, entonces, que la estimación de los resultados puede estar sujeta a la presencia de heterocedasticidad y autocorrelación, por lo que la metodología de mínimos cuadrados ordinarios (MCO) no es la más eficiente (Pérez, 2008, pp. 276-280).

En ese orden de ideas, y partiendo de una especificación general de la forma:

$$
y_{i t}=\beta_{0}+\sum_{k=1}^{k} \beta_{k} \chi_{k i t}+u_{i t}(10)
$$

con $\mathrm{k}$ representando el número de variables independientes, se pueden identificar varios tipos de modelos según la relación esperada entre las $k$ y la variable dependiente del panel $^{14}$. Una primera especificación conocida como el Modelo de Coeficientes Constantes supone que el $\beta_{k}$ estimado es invariable en ambas dimensiones, con lo cual se posibilita el uso de la metodología MCO si el término de error es homocedástico $\left(\operatorname{Var}\left[u_{i}\right]=\sigma^{2}\right)$ y no se presenta autocorrelación en su estructura $\left(\operatorname{Cov}\left[u_{i t}, u_{j \mathrm{j}}\right]=0 \forall i \neq j, \mathrm{y} \forall t \neq s\right)$. Este es un caso poco realista en la práctica, por lo que la ecuación (11) puede transformarse para incluir heterocedasticidad en $i$, correlación transversal y/o serial. En esta situación se suele utilizar la metodología de mínimos cuadrados generalizados (MCG) para controlar la heterocedasticidad observada o, en su defecto, la metodología de máxima verosimilitud para determinar la naturaleza del proceso autorregresivo.

En ese sentido, el modelo conocido como De Efectos Fijos incorpora a su estructura una serie de variables dicótomas para recoger los efectos de las variaciones temporales y/o transversales propias de las relaciones entre variables económicas (en términos de la ecuación 10, los efectos fijos se incorporan como un componente determinístico en $\tau_{i}$ y $\theta_{t}$ ). La ecuación de estimación se puede escribir como (Op. cit., p. 283):

$$
y_{i t}=\beta_{0}+\sum_{i=1}^{N-1} \tau_{i} d_{i}+\sum_{t=1}^{T-1} \theta_{t} g_{t}+\sum_{k=1}^{k} \beta_{k} \chi_{k i t}+u_{i t}+\varepsilon_{i t}(11)
$$

En la ecuación, $d_{i}$ y $g_{i}$ representan las nuevas variables dicótomas, una para cada $i$ y para cada $t$. También es posible incorporar en la ecuación de panel a las componentes $\tau_{i}$ y $\theta_{t}$, permitiendo que varíen estocásticamente, de tal manera que $\gamma_{i}$ sea específico para cada $\mathrm{i}$ (between o entre grupos), y $\theta_{t}$ ahora sea representativo para cada $t$ (within o intra grupos). Este tipo de modelos se conocen como De Efectos Aleatorios y requieren del uso de la metodología MCG.

No existe una regla general para seleccionar el modelo más adecuado para cada caso, sin embargo, se recomienda el uso de una especificación de efectos fijos cuando "el número de unidades de análisis (o de instantes en el tiempo) no sea grande" (Op. cit., p. 284), tal y como ocurre con la disponibilidad estadística de i en esta investigación.

14 Mayorga y Muñoz (2000) ofrecen información útil sobre los diversos modelos de panel de datos según su especificación y aplicación potencial. 
Después de seleccionar el modelo para el contraste de la PPA, se sugiere la realización de las pruebas de estacionariedad en panel (pruebas de raíz unitaria) para determinar la presencia de efectos temporales convergentes o divergentes en las series. La base analítica es muy similar a la que se utiliza para contrastar las series individuales, e incluye desarrollos de Maddala \& Wu (MW; 1996, 1999), Levin, Lin \& Chu (LLC, 2002), y de Im, Pesaran \& Shin (IPS; 2003); estos trabajos han perfeccionado las pruebas que fueron propuestas desde 1992 por Levin \& Lin. Primeramente, se encuentra el test MW, no paramétrico y no asintótico, que no requiere de un panel balanceado; además, se pueden utilizar diferentes extensiones de rezago en las pruebas ADF individuales (Maddala, 1999, p. 440).

Por su parte, LLC presenta un modelo mejorado para contrastar por raíz unitaria en paneles asintóticos con muestras finitas, de tal manera que la forma más general de la ecuación de regresión $\mathrm{ADF}$ es:

$$
\Delta y_{i t}=\alpha_{0 i}+\alpha_{1 i} \mathrm{t}+\delta y_{i t-1}+\varepsilon_{i t}(12)
$$

En la ecuación, $-2<\delta \leq 0$ para $i=1, \ldots, \mathcal{N}$. $\varepsilon_{i t}$ se distribuye independientemente a través de $i$, y sigue un proceso estacionario ARMA para cada $i$. Bajo la hipótesis nula $\delta=0 \mathrm{y}$ $\alpha_{1 i}=0 \forall i$, bajo la hipótesis alternativa $H_{1}: \delta<0$ y $\alpha_{1 i} \in \mathbb{R}$ (Levin et al., 2002, pp. 4-8). Como los mismos autores reconocen, el test planteado tiene la limitación de asumir que todos los $i$ "son idénticos con respecto a la presencia o ausencia de una raíz unitaria", condición difícil de explicar empíricamente (Op. cit., p. 18).

Precisamente, el test IPS se desarrolló buscando solventar la limitación derivada del test LLC. La formulación general es similar a la de la ecuación (13), dado que también se basa en una enunciación de tipo ADF; no obstante, la hipótesis alternativa se relaja para permitir que el coeficiente $\delta$ difiera entre grupos, por lo que no todos los individuos deben ser estacionarios bajo la $H_{1}$ (Im et al., 2003, p. 55).

\subsection{Cointegración en panel de datos}

Según Ramajo (2007, p. 24), las técnicas de cointegración en panel “...mejoran considerablemente las propiedades estadísticas de los contrastes [estándares] utilizados en el análisis de series temporales individuales, a la vez que se permite(n) un mayor grado de heterogeneidad tanto en los parámetros como en la dinámica temporal de las series implicadas". Entre los contrastes más utilizados para el tratamiento de paneles de datos se encuentra el formulado por Pedroni $(1999,2004)$ y el formulado por Maddala \& Wu (1999), basado en Fisher-Johansen.

El primero de ellos se fundamenta en los residuos estimados de la relación de cointegración analizada. Pedroni ha desarrollado siete tipos de estadísticos de cointegración basados en los residuos MCO de las ecuaciones de largo plazo, cuya hipótesis nula es 
la de no cointegración. Cuatro de estos contrastes siguen la línea de los estadísticos de panel (within-dimensión), mientras que los tres restantes van emparentados con los de promedio de grupo (between-dimensión). En ambos casos, los estadísticos estandarizados presentan una distribución asintótica N(0,1) (Ramajo, 2007, pp. 24-25; Pedroni, 2004).

Por su parte, el segundo contraste de cointegración desarrollado por Maddala \& Wu sigue a Fisher al proponer la construcción de estadísticos de panel bajo el enfoque multivariante de Johansen (1988, 1991), el cual permite la existencia de múltiples relaciones cointegradas entre las variables analizadas, a diferencia del test de Pedroni, que solamente admite la existencia de un vector de cointegración entre las variables por contrastar (Ramajo, 2007).

Finalmente, es importante anotar que ambos contrastes asumen el mismo grado de heterogeneidad individual, puesto que permiten que los coeficientes de cada relación de cointegración varíen de forma libre para cada unidad de estudio.

\section{La evidencia}

En este apartado se presentan los resultados econométricos en el siguiente orden: primero, las pruebas de raíces unitarias en panel; seguidamente, las posibles relaciones de cointegración para las variables del panel y los test de causalidad tipo Granger; por último, el mejor modelo por estimar según consideraciones teóricas y estadísticas.

\subsection{Contrastes de raíces unitarias en panel}

Como primera medida se estableció el orden de integración de las series ITCR, TCN e IPC en panel (todas en logaritmos), buscando evidencia para determinar su reversión a la media a largo plazo. En el anexo 2 se pueden observar los gráficos de las medias de las variables con una banda de más o menos una desviación estándar; se destaca el aplanamiento sustancial de la serie LOGIPC, especialmente después del ajuste generalizado de los años noventa ${ }^{15}$. Otro aspecto destacado es el de la reducción de la volatilidad de la serie LOGITCR para el mismo periodo; de hecho, hasta principios de los años noventa, los movimientos de las tasas reales estaban muy influenciados por los periodos de inflación elevada que sufrieron varios países latinoamericanos, siendo los casos más aberrantes los de Chile, bajo el Gobierno de Allende en la primera mitad de los setenta, así como el argentino, el brasileño y el peruano a finales de los años ochenta y comienzos de los noventa ${ }^{16}$. También debe destacarse el difícil escenario de reconstrucción de la serie LOGTCN, cuyo comportamiento parece estar dominado por las constantes

15 Con la posible excepción de Venezuela, que ha seguido un proceso de desarrollo particular desde finales de los años noventa.

16 Todos ellos caracterizados por periodos amplios de elevada inflación antes de las hiperinflaciones (Reinhart \& Savastano, 2003). 
fluctuaciones que se presentaron en muchos países en los años setenta, ochenta e incluso los noventa, con el efecto tequila y el efecto dragón, entre otras fuentes de volatilidad.

En la tabla 1 se recogen los principales resultados de los test de raíz unitaria en panel. Se observa que la hipótesis nula no se puede rechazar para el caso de las series originales; se rechaza en pocos casos al incorporar intercepto y tendencia, y también se rechaza con estadísticos más robustos en todos los casos en primeras diferencias, lo cual sugiere que las series son $\mathrm{I}(1)$, por lo tanto, se puede proceder al siguiente análisis.

\subsection{Contrastes de cointegración en panel}

A continuación, se realizaron las pruebas de cointegración MW y Pedroni, cuyos resultados se resumen en las tablas 2 y 3 , respectivamente. Los resultados Fischer-Johansen apuntan a que hay evidencia de la existencia de una relación de largo plazo entre las variables LOGITGR, LOGTGN y LOGIPG, lo que está acorde con lo que sugieren los desarrollos más recientes acerca de la PPA; tanto el test de traza como el test de rango indican que existen al menos dos ecuaciones de cointegración ${ }^{17}$. Por su parte, la prueba basada en Engle \& Granger reafirma la presencia de cointegración en una porción significativa de las unidades de corte transversal a través del tiempo.

Tabla 1. Test de raíz unitaria en panel

\begin{tabular}{|c|c|c|c|c|c|}
\hline \multicolumn{2}{|c|}{$\begin{array}{c}\text { LLG } \\
\text { H0: se asume un proceso de raíz } \\
\text { unitaria común. }\end{array}$} & \multicolumn{2}{|c|}{$\begin{array}{c}\text { IPS } \\
\text { H0: se asume un proceso de raíz } \\
\text { unitaria individual. }\end{array}$} & \multicolumn{2}{|c|}{$\begin{array}{l}\text { Maddala-Wu ADF Fisher Chi } \\
\text { cuadrado. H0: se asume un pro- } \\
\text { ceso de raíz unitaria individual. }\end{array}$} \\
\hline \multicolumn{2}{|c|}{ none } & \multicolumn{2}{|c|}{ none } & \multicolumn{2}{|c|}{ none } \\
\hline \multicolumn{6}{|c|}{ LOGITCR } \\
\hline Statistic & -1.41817 & Statistic & --- & Statistic & 10.3544 \\
\hline [Prob] & {$[0.3379]$} & [Prob] & --- & [Prob] & {$[0.8475]$} \\
\hline \multicolumn{6}{|c|}{ LOGTCN } \\
\hline Statistic & 6.14563 & Statistic & --- & Statistic & 16.0329 \\
\hline [Prob] & {$[1.0000]$} & [Prob] & --- & [Prob] & {$[0.4507]$} \\
\hline \multicolumn{6}{|c|}{ LOGIPG } \\
\hline Statistic & -1.20803 & Statistic & --- & Statistic & 22.9556 \\
\hline [Prob] & [0.1135] & [Prob] & --- & [Prob] & [0.1 149$]$ \\
\hline Individ & and trend & Individ & nd trend & Individ & $\mathrm{t}$ and trend \\
\hline
\end{tabular}

17 Se asume que el proceso presenta tendencia determinista lineal, si bien los resultados no difieren demasiado cuando se asume tendencia no lineal. 


\begin{tabular}{|c|c|c|c|c|c|}
\hline \multicolumn{6}{|c|}{ LOGITCR } \\
\hline $\begin{array}{l}\text { Statistic } \\
\text { Prob }\end{array}$ & $\begin{array}{c}-2.20106 \\
{[0.0139]^{* *}}\end{array}$ & $\begin{array}{l}\text { Statistic } \\
\text { [Prob] }\end{array}$ & $\begin{array}{l}-4.29310 \\
{[0.0000]^{*}}\end{array}$ & $\begin{array}{l}\text { Statistic } \\
{[\text { Prob] }}\end{array}$ & $\begin{array}{c}56.3071 \\
{[0.0000]^{*}}\end{array}$ \\
\hline \multicolumn{6}{|c|}{ LOGTGN } \\
\hline $\begin{array}{l}\text { Statistic } \\
\text { [Prob] }\end{array}$ & $\begin{array}{l}2.29443 \\
{[0.9891]}\end{array}$ & $\begin{array}{l}\text { Statistic } \\
\text { [Prob] }\end{array}$ & $\begin{array}{l}2.42398 \\
{[0.9923]}\end{array}$ & $\begin{array}{l}\text { Statistic } \\
{[\text { Prob] }}\end{array}$ & $\begin{array}{c}22.6052 \\
{[0.1247]}\end{array}$ \\
\hline \multicolumn{6}{|c|}{ LOGIPG } \\
\hline $\begin{array}{l}\text { Statistic } \\
\text { [Prob] }\end{array}$ & $\begin{array}{c}-1.28779 \\
{[0.0989]}\end{array}$ & $\begin{array}{l}\text { Statistic } \\
\text { [Prob] }\end{array}$ & $\begin{array}{c}-1.54778 \\
{[0.0608]}\end{array}$ & $\begin{array}{l}\text { Statistic } \\
{[\text { Prob }]}\end{array}$ & $\begin{array}{c}110.981 \\
{[0.0000]^{*}}\end{array}$ \\
\hline \multicolumn{2}{|c|}{ Primeras diferencias } & \multicolumn{2}{|c|}{ Primeras diferencias } & \multicolumn{2}{|c|}{ Primeras diferencias } \\
\hline \multicolumn{6}{|c|}{ LOGITCR } \\
\hline $\begin{array}{l}\text { Statistic } \\
\text { [Prob] }\end{array}$ & $\begin{array}{l}-91.6881 \\
{[0.0000]^{*}}\end{array}$ & $\begin{array}{l}\text { Statistic } \\
\text { [Prob] }\end{array}$ & $\begin{array}{l}-61.0393 \\
{[0.0000]^{*}}\end{array}$ & $\begin{array}{l}\text { Statistic } \\
{[\text { Prob }]}\end{array}$ & $\begin{array}{c}1541.06 \\
{[0.0000]^{*}}\end{array}$ \\
\hline \multicolumn{6}{|c|}{ LOGTGN } \\
\hline $\begin{array}{l}\text { Statistic } \\
\text { [Prob] }\end{array}$ & $\begin{array}{l}-70.0187 \\
{[0.0000]^{*}}\end{array}$ & $\begin{array}{l}\text { Statistic } \\
\text { [Prob] }\end{array}$ & $\begin{array}{l}-47.8543 \\
{[0.0000]^{*}}\end{array}$ & $\begin{array}{l}\text { Statistic } \\
{[\text { Prob] }}\end{array}$ & $\begin{array}{c}1128.04 \\
{[0.0000]^{*}}\end{array}$ \\
\hline \multicolumn{6}{|c|}{ LOGIPC } \\
\hline $\begin{array}{l}\text { Statistic } \\
\text { [Prob] }\end{array}$ & $\begin{array}{l}-9.14093 \\
{[0.0000]^{*}}\end{array}$ & $\begin{array}{l}\text { Statistic } \\
\text { [Prob] }\end{array}$ & $\begin{array}{l}-9.46095 \\
{[0.0000]^{*}}\end{array}$ & $\begin{array}{l}\text { Statistic } \\
{[\text { Prob] }}\end{array}$ & $\begin{array}{c}129.339 \\
{[0.0000]^{*}}\end{array}$ \\
\hline
\end{tabular}

Fuente: Cálculos del autor con Eviews 6.0.

Hasta aquí los resultados están acordes con la evidencia internacional que explica la existencia de una relación de largo plazo entre las variables nominales que conforman la TCR. En un trabajo posterior se propone la realización de un análisis de cambio estructural diferenciado por periodo pre y posapertura, para confrontar así el efecto de reversión a la media.

Tabla 2. Test de cointegración en panel de Fisher-Johansen de Maddala \& Wu

\begin{tabular}{|c|c|c|c|c|}
\hline \multicolumn{5}{|c|}{ H0: no cointegración } \\
\hline Hipothesized N ${ }^{\circ}$ of $\mathrm{CE}(\mathrm{s})$ & Trace statistic & Prob & Max-eigen statistic & Prob \\
\hline $\mathrm{r}=0$ & $194.4^{*}$ & 0.0000 & $179.9 *$ & 0.0000 \\
\hline $\mathrm{r} \leq 1$ & $65.55 *$ & 0.0000 & $54.09 *$ & 0.0000 \\
\hline $\mathrm{r} \leq 2$ & $37.52^{*}$ & 0.0018 & $37.52^{*}$ & 0.0018 \\
\hline
\end{tabular}

Fuente: Cálculos del autor con Eviews 6.0. 
Tabla 3. Test de cointegración en panel de Pedroni basado en Engle \& Granger

\begin{tabular}{|c|c|c|c|c|}
\hline \multicolumn{5}{|c|}{ Hipótesis nula: no cointegración } \\
\hline & v-statistic & rho-statistic & PP-statistic & ADF-statistic \\
\hline \multicolumn{5}{|c|}{ Weighted panel stats (within) } \\
\hline Statistic & -3.154568 & -2.887337 & -4.211529 & -5.983461 \\
\hline [Prob] & {$[0.9992]$} & {$[0.0019]^{*}$} & {$[0.0000]^{*}$} & {$[0.0000]^{*}$} \\
\hline \multicolumn{5}{|c|}{ Group-mean stats (between) } \\
\hline Statistic & - & 1.374309 & -2.340790 & -2.551136 \\
\hline [Prob] & - & {$[0.9153]$} & {$[0.0096]^{*}$} & {$[0.0054]^{*}$} \\
\hline
\end{tabular}

Fuente: Cálculos del autor con Eviews 6.0.

Seguidamente se hicieron test de causalidad en el sentido de Granger, con el fin de determinar la existencia de causalidad bidireccional entre las series y sus efectos temporales (tabla 4). En efecto, se pudo observar que hay evidencia de la presencia de causalidad Granger bidireccional a medida que la extensión del rezago escogido aumenta, desde un trimestre para el caso de las variables LOGTCN y LOGIPC, dos trimestres para las variables LOGITGR e LOGIPG, hasta 5 trimestres inclusive para las variables LOGITGR y LOGTCN, con un nivel de significancia del $95 \%$. Este resultado plantea el problema de la velocidad de ajuste de las variables nominales que afecta a una variable real y que determina su reversión a la media. Es notable el caso de la relación entre LOGITCR y LOGTGN, en la que teóricamente se esperaría un efecto de causalidad más robusto e inmediato. El tiempo que toma dicha reversión es tema para una investigación posterior. Adicionalmente, la relación de causalidad bidireccional LOGTGN y LOGIPG sugiere que el efecto de trasmisión de los precios relativos es relativamente rápido, aunque no se cuenta con información específica para bienes transables y no transables.

Tabla 4. Test de causalidad Granger

\begin{tabular}{|c|c|c|}
\hline Ho: no causalidad en el sentido de Granger & F & Prob \\
\hline LOGITCR y LOGIPG & 13.6399 & 0.0000 \\
\hline LOGIPG y LOGITCR & 1.82666 & 0.0388 \\
\hline LOGTGN y LOGIPG & 7.90029 & 0.0000 \\
\hline LOGIPG y LOGTCN & 319.886 & 0.0000 \\
\hline LOGTCN y LOGITCR & 1.64996 & 0.0716 \\
\hline LOGITCR y LOGTCN & 2.93534 & 0.0005 \\
\hline
\end{tabular}

Rezago especificado: 12 
La última etapa de este trabajo consistió en la búsqueda de un modelo de predicción para la PPA de acuerdo con los hechos estilizados propuestos por Froot \& Rogoff (1994), Rogoff (1996), Obstfeld \& Rogoff (2000), Sarno \& Taylor (2002) y Frankel (2006), entre otros autores. Se asume un panel heterocedástico con estimadores de efectos fijos para contrastar la hipótesis fuerte de la PPA, tal como se expresa a continuación ${ }^{18}$ :

$$
\operatorname{LOGITCR}_{i t}=\beta_{0 i}+\beta_{1 i t} \operatorname{LOGTCN}_{i t}+\beta_{2 i t} \operatorname{LOGIPC}_{i t}+\mu_{i t}(13)
$$

En la ecuación, LOGITCR $_{i t}$ representa el diferencial de precios de cada unidad de sección cruzada con referencia a Colombia. Los resultados presentados en la tabla 5 no muestran la robustez general esperada, y vislumbran un problema de autocorrelación normalmente asociado al efecto de heterocedasticidad. Más aún, el signo del coeficiente de la variable LOGTCN no es el que se espera teóricamente. Este último resultado, sin embargo, puede deberse a una mezcla de problemas que van desde la calidad de las estadísticas históricas latinoamericanas (Mahía, 2000, p. 6), pasando por lo variopinto de los regímenes cambiarios, hasta la carencia de otras variables explicativas importantes, como ya se mencionó. Un aspecto destacable de los resultados es que el coeficiente de la variable LOGTCN es mayor que el de la variable LOGIPC; esto se explica por el hecho de que, en la práctica, la velocidad de ajuste de la ton es más rápida que la de los precios relativos medidos por IPC.

A manera de corolario, se puede decir que no existe evidencia definitiva que permita establecer el cumplimiento de la PPA en el caso de Latinoamérica. Sin embargo, en futuros escenarios se hará necesario explorar otras opciones teóricas y metodológicas que permitan avanzar en el entendimiento de las relaciones entre los precios y los tipos de cambio con el fin de que los encargados de la política económica tengan mejores instrumentos para la toma de decisiones.

18 Según resultados de la prueba de Hausman que se muestran en el anexo 4. 
Tabla 5. Resultados econométricos estimación panel EGLS

\begin{tabular}{|c|c|c|c|c|c|}
\hline \multicolumn{2}{|c|}{ Variable dependiente LOGITCR } & & & \multirow{5}{*}{ Prob } & \multirow{5}{*}{$\begin{array}{l}\text { Std. } \\
\text { Error }\end{array}$} \\
\hline \multicolumn{2}{|c|}{ Unidades de corte transversal 8} & \multirow{4}{*}{ Coefficient } & \multirow{4}{*}{ t-Statistic } & & \\
\hline Periodos 539 & & & & & \\
\hline \multicolumn{2}{|c|}{$\begin{array}{l}\text { White cross-section standard errors \& } \\
\text { covariance (no d.f. correction) }\end{array}$} & & & & \\
\hline \multicolumn{2}{|c|}{ Effects Cross-section fixed (dummy variables) } & & & & \\
\hline \multicolumn{2}{|c|}{$\mathrm{C}$} & 4.818971 & 344.3237 & 0.0000 & 0.013995 \\
\hline \multicolumn{2}{|c|}{ LOGTCN } & -0.022506 & -7.201151 & 0.0000 & 0.003125 \\
\hline \multicolumn{2}{|c|}{ LOGIPG } & -0.012603 & -7.823596 & 0.0000 & 0.001611 \\
\hline R-squared & 0.226846 & & & & \\
\hline Adjusted R-squared & 0.224514 & & & & \\
\hline F-statistic & 97.24718 & & & & \\
\hline Prob(F-statistic) & 0.000000 & & & & \\
\hline Durbin-Watson stat & 0.040832 & & & & \\
\hline
\end{tabular}

\section{Conclusiones}

El principal resultado que deriva de esta investigación es que no hay evidencia concluyente que permita inferir que para los países latinoamericanos de referencia se cumple la PPA. Sin embargo, esto no significa que dicha teoría no aplique para la región, sino, más bien, que se deben explorar otros escenarios de trabajo para contrastar la hipótesis de forma más adecuada a las condiciones de las economías regionales. Como ya se mencionó, algunos modelos para la TCR incluyen correcciones que presuponen desviaciones permanentes debido a movimientos de sus fundamentales; en estos modelos, la dinámica del ajuste se constituye en un instrumento fundamental para entender los efectos que los cambios de dichos fundamentales tienen en los desequilibrios de la TCR.

Este documento representa un esfuerzo exploratorio complejo que se constituye en un buen punto de partida para futuras investigaciones que ayuden a comprender mejor el problema de la trasmisión de los precios entre países en un mundo cada vez más interconectado en términos comerciales y financieros. 


\section{Referencias}

Al Omar, M., \& Ghali, K. (2009). PPP Tests in Cointegrated Heterogeneous Panels: The Case of the GCG Countries. International Research Journal of Finance and Economics, 35, 163-172.

Alonso, G., Hernández, J., Pulido, J. y Villa, M. (2008). Medidas alternativas de tasa de cambio real para Colombia. Borradores de Economía, 514. Recuperado de http:/ /www. banrep.gov.co/docum/ftp/borra514.pdf

Arango, F., \& Meisel, A. (1994). Comentarios al artículo "Índices de precios y deflactores de la tasa de cambio". Coyuntura Económica, XXIV(4), 157-164.

Azali, M., Habibullah, M.S., \& Baharumshah, A.Z. (2001). Does PPP hold between asian and japanese economies? Evidence using panel unit root and panel cointegration. Japan and the World Economy, 13(1), 35-50. DOI:10.1016/S0922-1425(00)00055-4

Bergin, P., Glick, R., \& Wu, J. (2013). The micro-macro disconnect of purchasing power parity. The Review of Economics and Statistics, 95(3), 798-812.

Carrasquilla, A. (1989). Minidevaluaciones y paridad en el poder adquisitivo: el caso de Colombia. Desarrollo y Sociedad, 23, 69-82.

Choi, I., \& Chue, T. (2007). Subsampling hypothesis tests for nonstationary panels with applications to exchange rates and stock prices. Fournal of Applied Econometrics, 22(2), 233-264.

Chortareas, G., \& Kapetanios, G. (2009). Getting PPP right: Identifying mean-reverting real exchange rates in panels. Fournal of Banking and Finance, 33(2), 390-404.

Cheung, Y., Chinn, M., \& Fujii, E. (2009). Pitfalls in measuring exchange rate misalignment: The yuan and other currencies. Open Economies Review, 20(2), 183-206.

Dickey, D., \& Fuller, W. (1979). Distribution of the estimators for autoregressive time series with a unit root. Fournal of the American Statistical Association, 74(366), 427-431.

Dornbusch, R. (1988). Purchasing Power Parity. En Palgrave Macmillan, The New Palgrave Dictionary of Economics (pp. 1075-1084). Nueva York: Stockton Press. - (1993). La macroeconomía de una economía abierta. Antoni Bosch Editor, Barcelona.

Echavarría, J., Vásquez, D. y Villamizar, M. (2005). La tasa de cambio real en Colombia. ¿Muy lejos del equilibrio? Borradores de Economía, 337. Recuperado de http://www. banrep.gov.co/docum/ftp/borra337.pdf 
Echavarría, J., López, E. y Misas, M. (2008). El tipo de cambio real de equilibrio en Colombia y su desalineamiento: Estimación a través de un modelo SVEG. Ensayos sobre Política Económica, 26(57), 282-319.

Engle, R. \& Granger, C, (1987). Cointegration and error correction: Representation, estimation and testing. Econometrica, 55(2), 251-276.

Frankel, J. (2006). The Balassa-Samuelson relationship and the Renminbi. Recuperado de https://www.hks.harvard.edu/fs/jfrankel/BalassaSamuelson\&ChinaRMB.pdf

Frenkel, J. (1981). The collapse of purchasing power parity during the 1970s. European Economic Review, XVI(1), 145-165.

Froot, K., \& Rogoff, K. (1994, diciembre). Perspectives on PPP and long run real exchange rates (n.o W4952). NBER Working Paper Series.

Froot, K., Kim, M., \& Rogoff, K. (2001, noviembre). The law of one price over 700 years (n.o 01/174). IMF Working Paper - Research Department. Recuperado de https://www. imf.org/external/pubs/ft/wp/2001/wp01174.pdf

Huertas, C. (2002). Tasa de cambio real: Definición, equilibrio y metodología de cálculo en Colombia. Reportes del Emisor, 40. Recuperado de http://www.banrep.gov.co/ sites/default/files/publicaciones/archivos/re_40.pdf

- (2005). Tasa de cambio real de Colombia: Un enfoque empírico no lineal. Borradores de Economía, 359. Recuperado de http://www.banrep.gov.co/docum/ftp/ borra359.pdf

Huertas, C. \& Oliveros, H. (2003). Desequilibrios nominales y reales del tipo de cambio en Colombia. Ensayos sobre Política Económica, 43, 32-65. Recuperado de http://www. banrep.gov.co/sites/default/files/publicaciones/archivos/espe_043-2.pdf

Im, K., Pesaran, H., \& Shin, Y. (2003). Testing for unit roots in heterogeneous panels. Journal of Econometrics, 115(1), 53-74.

Imbs, J., Mumtaz, H., Ravn, M., \& Rey, H. (2005). PPP strikes back aggregation and the real exchange rate. The Quarterly fournal of Economics, 120(1), 1-43.

Isard, P. (1977). How far can we push the law of one price? American Economic Reviewe, 67(5), 942-948.

Jenkins, M., \& Snaith, S. (2005). Tests of purchasing power parity via cointegration analysis of heterogeneous panels with consumer price indices. Fournal of Macroeconomics, $27(2), 345-362$. 
ISSN: 0124-3551 / Año 16, No 25 / julio-diciembre / pp. 123-152

Johansen, S. (1988), Statistical analysis of cointegrating vectors. Fournal of Economic Dynamics and Control, 12(2/3), 231-254.

. (1991), Estimation and Hypothesis Testing of Cointegrating Vectors in Gaussian Vector Autoregressive Models. Econometrica, 59(6), 1551-1580.

. (2002). A small sample correction for the Test of Cointegrating Rank in the Vector Autoregressive Model. Econometrica, 70(5), 1929-1961.

Kilian, L., \& Taylor, M. (2001, noviembre). Why is it so difficult to beat the random walk forecast of exchange rates? (n.o 88). European Central Bank - Working Paper Series. Recuperado de http://fordschool.umich.edu/rsie/workingpapers/Papers451-475/ r464.pdf

Krugman, P. (1978). Purchasing power parity and exchange rates: Another look at the evidence. Fournal of International Economics, 8(3), 397-407.

Krugman, P., \& Obstfeld, M. (2003). International economics: Theory and policy (6.a ed.). EE. UU.: Pearson Education. Recuperado de http://goo.gl/TLWSPF

Levin, A. \& Lin, C (1992). Unit Root Test in Panel Data: Asymptotic and Finite Sample Properties. Discussion paper, University of California, 92-93.

Levin, A., Lin, C., \& Chu, C. (2002). Unit root tests in panel data: Asymptotic and finite-sample properties. Fournal of Econometrics, 108, 1-24. Recuperado de http:// homepage.ntu.edu.tw/ clin/2002pur.pdf

Maddala, G.S. (1999). On the use of panel data method with cross-country data. Annales D’Économie et de Statistique, 55/56, 429-448.

Maddala, G.S., \& Wu, S. (1999). A comparative study of unit root Tests with Panel Data and a New Simple Test. Oxford Bulletin of Economics and Statistics, Especial issue, 631-652.

Mahía, R. (2000). Análisis de estacionariedad con datos de panel: Una ilustración para los tipos de cambio, precios y mantenimiento de la PPA en Latinoamérica. España: Instituto L. R. Klein. Recuperado de http://www.uam.es/otroscentros/klein/docjor/mahia.pdf

Mayorga, M. y Muñoz, E. (2000, septiembre). La técnica de datos de panel una guía para su uso e interpretación. Banco Central de Costa Rica. Documento de trabajo. Recuperado de http://goo.gl/Ht7u4Q

Meese, R., \& Rogoff, K. (1985, octubre). Was it real? The exchange rate -interest differential relation, 1973-1984 (n.o 1732). NBER Working Paper Series. 
Meisel, A. (1994). Como determinar si el peso está sobrevaluado o subvaluado. Borradores de Economía, 6.

Obstfeld, M., \& Rogoff, K. (2000, julio). The six mayor puzzles in international macroeconomics: Is there a common cause? (n.o W7777). NBER Working Paper Series.

Oliveros, H., \& Rowland, P. (2003). Colombian purchasing power parity analysed using a framework of multivariate cointegration. Borradores de Economía, 252.

Pedroni, P. (1999). Critical values for cointegration Tests in Heterogenous Panel with Multiple Regressors. Oxford Bulletin of Economics and Statistics, Especial issue, 653-670.

- (2001). Purchasing power parity tests in cointegrated panels. The Review of Economics and Statistics, 83(4), 727- 731. Recuperado de http://web.williams.edu/ Economics/wp/pedronipurchasing.pdf

- (2004). Panel cointegration: Asymptotic and finite sample properties of pooled time series tests with an application to the PPP hypothesis. Econometric Theory, 20, 597-625. Recuperado de http://web.williams.edu/Economics/wp/pedronipanelcointegration.pdf

Pérez, C. (2008). Econometría avanzada. Técnicas y herramientas. Madrid: Pearson - Prentice Hall.

Ramajo, J. (2007). Contraste empírico del modelo monetario del tipos de cambio: cointegracion y ajuste no lineal. Departamento de Economía, Universidad de Extremadura, 6. Recuperado de http://www.minhac.es/ief/principal.htm

Reinhart, G. \& Savastano, M. (2003). Realidades de las hiperinflaciones modernas. Finanzas y Desarrollo, junio, 20-23.

Rincón, H. (2000). Devaluación y precios agregados en Colombia, 1980-1998. Desarrollo y Sociedad, 46, 109-144. Recuperado de http://www.cemla.org/red/papers2000/v_ red_rincon.PDF

Rogoff, K. (1996). The purchasing power parity puzzle. Fournal of Economic Literature, 34(2), 647-668. Recuperado de http://www.yorku.ca/yildirim/ADMS4542/ PPPPuzzle.pdf

Rowland, P. (2003). Forecasting the USD/COP exchange rate: A random walk with a variable drift. Borradores de Economía, 254.

Sarno, L., \& Taylor, M. (2002). Purchasing power parity and the real exchange rate. IMF Staff Papers, 49(1), 65-105. 
ISSN: 0124-3551 / Año 16, No 25 / julio-diciembre / pp. 123-152

Steiner, R. \& Wüllner, A. (1994). Índices de precios y deflactores de la tasa de cambio. Coyuntura Económica, 24(2).

Summers, R., \& Heston, A. (1991). The Penn World Table (Mark 5): An expanded set of international comparisons, 1950-1988, Quarterly Fournal of Economics, 106(2), 327-68.

Taylor, A. (2000a, marzo). Potential pitfalls for the purchasing power parity puzzle? Sampling and specification biases in mean reversion tests of the law of one price (n.o W7577). NBER Working Paper Series.

. (2000b, noviembre). A century of purchasing power parity (n.o W8012). NBER Working Paper Series.

Wu, Y. (1996). Are real exchange rates nonstationary? Evidence from a Panel Data Test. Fournal of Money, Credit and Banking, 18(1), 54-63. Recuperado de http://goo.gl/ pS64BN 


\section{Anexos}

\section{Anexo 1. Descripción de las variables}

\begin{tabular}{|c|c|c|}
\hline & TGN & IPG \\
\hline Argentina & $\begin{array}{l}\text { Fuente: Banco Central de la República } \\
\text { Argentina. Anual con datos fragmentarios } \\
\text { hasta } 2001 \text { y mensual entre 2002-2014. }\end{array}$ & $\begin{array}{l}\text { Fuente: INDEC. Gran Buenos Aires, } \\
\text { serie mensual empalmada ,1970-2014, } \\
\text { base } 2013 \text {. }\end{array}$ \\
\hline Brasil & $\begin{array}{l}\text { Fuente: Banco Central do Brasil, } \\
\text { 1953-2014, mensual. }\end{array}$ & $\begin{array}{l}\text { Fuente: IBGE. Mensual, 1994-2014, } \\
\text { base } 2013 .\end{array}$ \\
\hline Ghile & $\begin{array}{l}\text { Fuente: Banco Central de Chile, } \\
\text { 1982-2014, mensual }\end{array}$ & $\begin{array}{l}\text { Fuente: INE. Serie histórica mensual } \\
\text { empalmada, 1928-2014, base } 2013 .\end{array}$ \\
\hline Ecuador & $\begin{array}{l}\text { Fuente: Banco Central de Ecuador, } \\
\text { 1925-2001 mensual (en marzo de } 2000 \\
\text { se inicia la dolarización). }\end{array}$ & $\begin{array}{l}\text { Fuente: INEC. Serie empalmada, } \\
\text { 1969-2014, mensual, base } 2004 .\end{array}$ \\
\hline México & $\begin{array}{l}\text { Fuente: Banco de México, 1954-2014, } \\
\text { mensual. }\end{array}$ & $\begin{array}{l}\text { Fuente: INEG - Banco de México, } \\
\text { 1969-2014, mensual, base } 2010 .\end{array}$ \\
\hline Perú & $\begin{array}{l}\text { Fuente: Banco Central de Reserva } \\
\text { del Perú, 1970-2014, mensual. }\end{array}$ & $\begin{array}{l}\text { Fuente: INEI. Lima, serie empalmada, } \\
\text { 1950-2014, mensual, base } 1994 .\end{array}$ \\
\hline Venezuela & $\begin{array}{l}\text { Fuente: Banco Central de Venezuela, } \\
\text { 1985-2014, mensual. }\end{array}$ & $\begin{array}{l}\text { Fuente: INE. Caracas, 1950-2014, } \\
\text { mensual, base } 2007 .\end{array}$ \\
\hline
\end{tabular}

\section{Anexo 2. Gráfico de medias de las variables en panel}

\section{LOG(ITCR)}

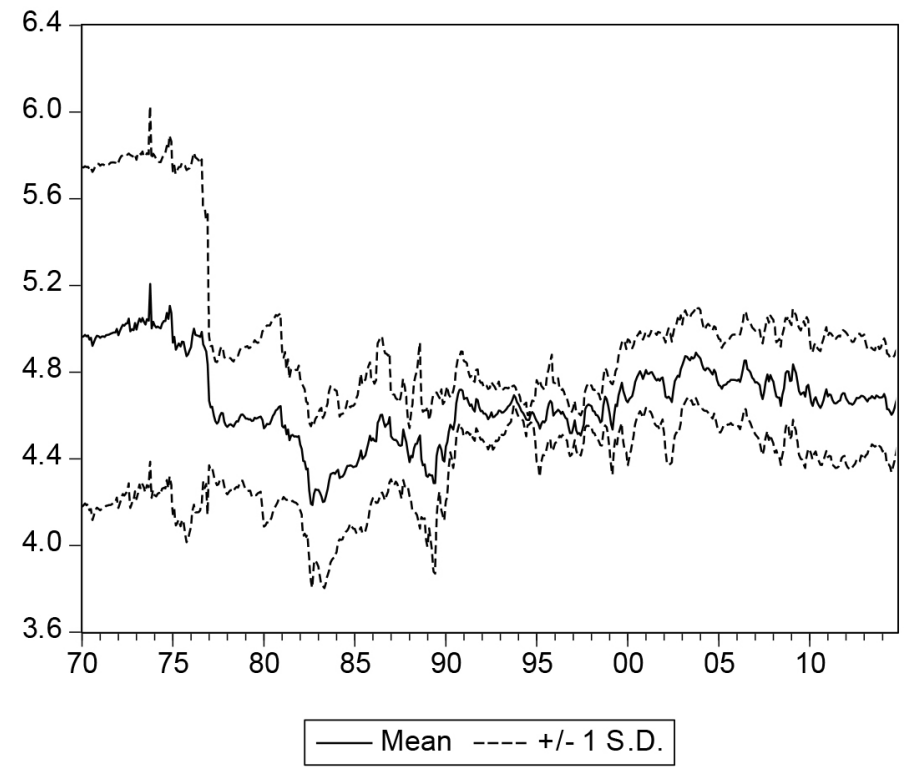




\section{CĆl'F́= 25}

ISSN: 0124-3551 / Año 16, No 25 / julio-diciembre / pp. 123-152

\section{LOG(TCN)}

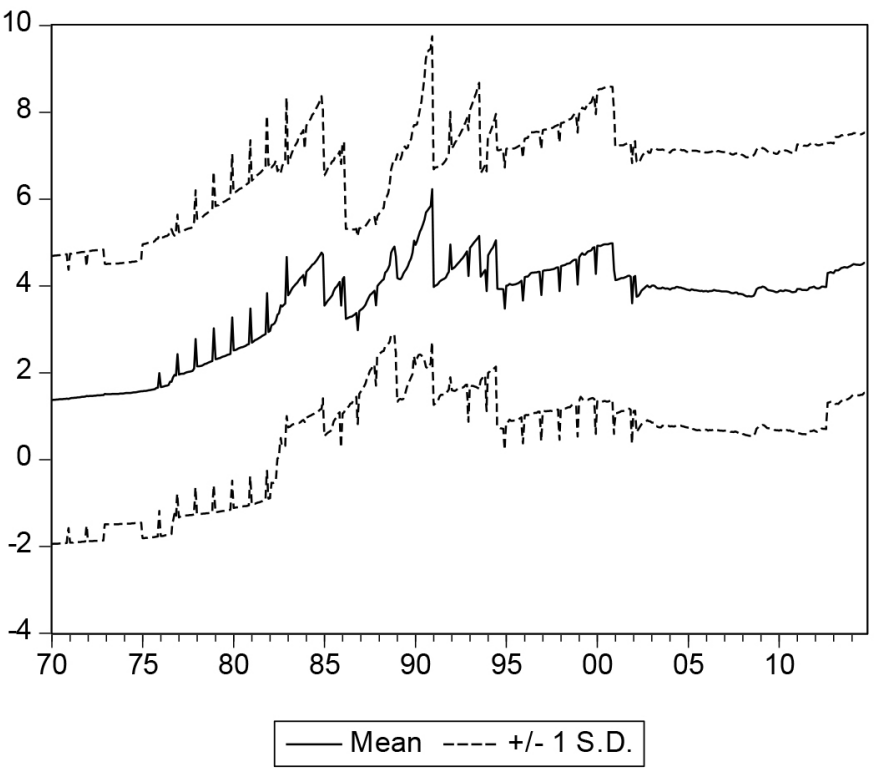

LOG(IPC)

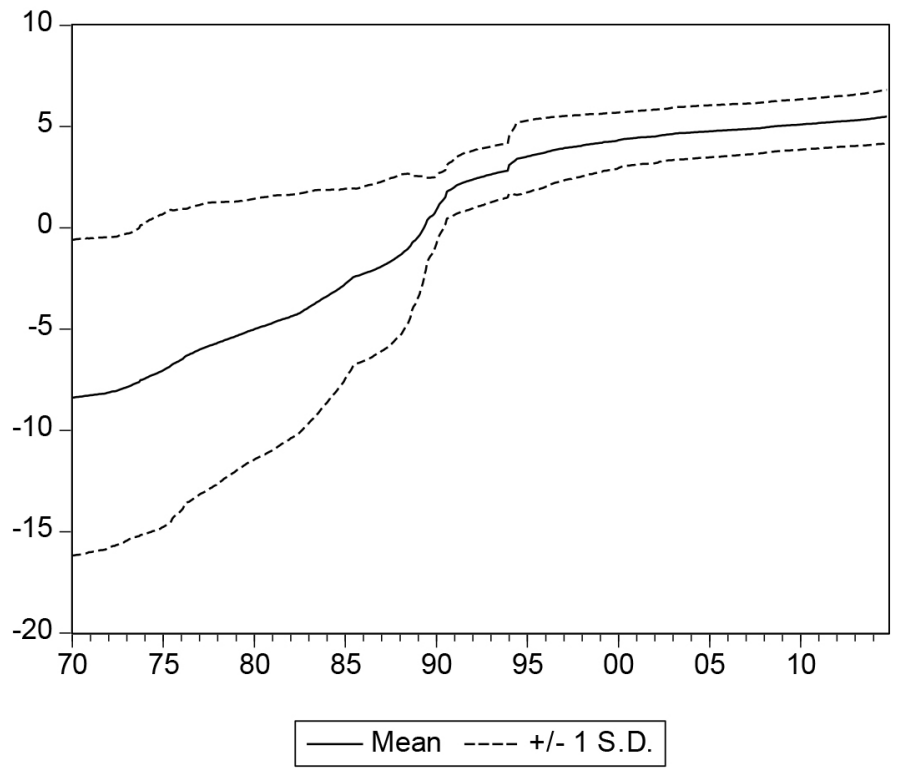




\section{Anexo 3. Resultados econométricos test de cointegración}

\section{Johansen Fisher Panel Gointegration Test}

Series: LOGIPG LOGITCR LOGTCN

Sample: 1970M01 2014M11

Included observations: 4312

Trend assumption: Linear deterministic trend

Lags interval (in first differences): 11

\section{Individual cross section results}

Trace Test

Max-Eign Test

\begin{tabular}{|c|c|c|c|c|}
\hline Cross Section & Statistics & Prob.** & Statistics & Prob.** \\
\hline \multicolumn{5}{|c|}{ Hypothesis of no cointegration } \\
\hline ARG & 61.3519 & 0.0000 & 29.7771 & 0.0024 \\
\hline BRA & 54.9352 & 0.0000 & 50.6607 & 0.0000 \\
\hline CHI & 111.0199 & 0.0000 & 78.2080 & 0.0000 \\
\hline COL & 55.0549 & 0.0000 & 45.2086 & 0.0000 \\
\hline ECU & 75.4622 & 0.0000 & 65.4440 & 0.0000 \\
\hline MEX & 65.8251 & 0.0000 & 46.7793 & 0.0000 \\
\hline PER & 140.8874 & 0.0001 & 118.5355 & 0.0001 \\
\hline VEN & 38.0881 & 0.0044 & 27.9375 & 0.0047 \\
\hline \multicolumn{5}{|c|}{ Hypothesis of at most 1 cointegration relationship } \\
\hline ARG & 31.5747 & 0.0001 & 28.9324 & 0.0001 \\
\hline BRA & 4.2746 & 0.8802 & 4.1672 & 0.8413 \\
\hline CHI & 32.8119 & 0.0001 & 22.4200 & 0.0021 \\
\hline COL & 9.8463 & 0.2927 & 8.8560 & 0.2983 \\
\hline ECU & 10.0182 & 0.2793 & 7.7816 & 0.4013 \\
\hline MEX & 19.0458 & 0.0140 & 12.6937 & 0.0872 \\
\hline PER & 22.3520 & 0.0040 & 20.3569 & 0.0048 \\
\hline VEN & 10.1507 & 0.2693 & 10.1392 & 0.2030 \\
\hline \multicolumn{5}{|c|}{ Hypothesis of at most 2 cointegration relationship } \\
\hline ARG & 2.6424 & 0.1040 & 2.6424 & 0.1040 \\
\hline BRA & 0.1074 & 0.7431 & 0.1074 & 0.7431 \\
\hline CHI & 10.3920 & 0.0013 & 10.3920 & 0.0013 \\
\hline $\mathrm{COL}$ & 0.9903 & 0.3197 & 0.9903 & 0.3197 \\
\hline ECU & 2.2366 & 0.1348 & 2.2366 & 0.1348 \\
\hline MEX & 6.3522 & 0.0117 & 6.3522 & 0.0117 \\
\hline PER & 1.9950 & 0.1578 & 1.9950 & 0.1578 \\
\hline VEN & 0.0115 & 0.9145 & 0.0115 & 0.9145 \\
\hline **MacKinnon-H & nelis (199؛ & & & \\
\hline
\end{tabular}




\section{Pedroni Residual Cointegration Test}

\section{Series: LOGIPG LOGITCR LOGTCN}

Sample: 1970M01 2014M11

Included observations: 4312

Cross-sections included: 8

Trend assumption: No deterministic trend

Lag selection: Automatic SIC with max lag of 14 to 18

Newey-West bandwidth selection with Bartlett kernel

Cross section specific results

Phillips-Peron results (non-parametric)

\begin{tabular}{|c|c|c|c|c|c|}
\hline Cross ID & AR $(1)$ & Variance & HAC & Bandwidth & Obs \\
\hline ARG & 0.910 & 0.455311 & 0.724968 & 3.00 & 176 \\
\hline BRA & 0.883 & 0.006566 & 0.011043 & 3.00 & 250 \\
\hline CHI & 0.984 & 0.001351 & 0.001351 & 0.00 & 389 \\
\hline COL & 0.989 & 0.000746 & 0.000745 & 11.00 & 335 \\
\hline ECU & 0.971 & 0.015428 & 0.015428 & 0.00 & 371 \\
\hline MEX & 0.954 & 0.006185 & 0.005079 & 2.00 & 538 \\
\hline PER & 0.964 & 2.808357 & 2.571332 & 14.00 & 538 \\
\hline VEN & 0.968 & 0.004998 & 0.006126 & 6.00 & 358 \\
\hline
\end{tabular}

Augmented Dickey-Fuller results (parametric)

\begin{tabular}{|c|c|c|c|c|c|}
\hline Cross ID & AR(1) & Variance & Lag & Max lag & Obs \\
\hline ARG & 0.892 & 0.387452 & 1 & 14 & 174 \\
\hline BRA & 0.912 & 0.005509 & 1 & 15 & 249 \\
\hline CHI & 0.978 & 0.001127 & 1 & 16 & 388 \\
\hline COL & 0.986 & 0.000733 & 1 & 16 & 334 \\
\hline ECU & 0.971 & 0.015428 & 0 & 16 & 371 \\
\hline MEX & 0.963 & 0.005889 & 4 & 18 & 534 \\
\hline PER & 0.964 & 2.808357 & 0 & 18 & 538 \\
\hline VEN & 0.962 & 0.004872 & 1 & 16 & 357 \\
\hline
\end{tabular}

\section{Anexo 4. Resultados econométricos test de Hausman}

\begin{tabular}{|l|c|c|}
\hline \multicolumn{3}{c}{ Test de Hausman para efectos fijos - aleatorios } \\
\hline \\
\hline
\end{tabular}

